\title{
Caracterización morfológica de la gallina criolla de traspatio de la subregión Sabana departamento de Sucre (Colombia)
}

\author{
Donicer Montes $\mathrm{V}^{* 1} \bowtie(0)$ Ph.D; Jaime de la Ossa V1 $₫\left(\mathbb{0}\right.$ Ph.D; Darwin Hernández $\mathrm{H}^{1} \bowtie \mathbb{0}$ Ph.D.
}

${ }^{1}$ Universidad de Sucre, Facultad de Ciencias Agropecuarias, Sincelejo, Colombia.

*Correspondencia: donicer.montes@unisucre.edu.co

Recibido: Noviembre 2018; Aceptado: Marzo 2019; Publicado: Mayo 2019.

\section{RESUMEN}

Objetivo. Caracterizar morfométrica y faneróptica de la gallina Criolla de traspatio de la subregión Sabana, departamento de Sucre (Colombia). Materiales y métodos. Se evaluaron 520 animales adultos criollos (350 gallinas y 170 gallos), en el periodo 2017-2018, ubicados en 10 localidades de la subregión Sabanas del departamento de Sucre Colombia. Se recolectó la información en un formato de encuesta integrado por variables cuantitativas y cualitativas. Los datos obtenidos fueron sometidos a análisis descriptivos univariado para muestras independiente. Además, se empleó la técnica de análisis de varianza. Las comparaciones entre machos y hembras se realizaron mediante una prueba de t. Los análisis fueron realizados usando paquete estadístico R. Resultados. Los descriptores analizados mostraron superioridad de los gallos sobre las gallinas $(p<0.05)$. El análisis de varianza en la población de hembras con respecto al peso, determinó que existen diferencias significativas $(p<0.05)$. Las características morfológicas y fanerópticas estudiadas, describen un ave de metatarso amarillo, con color de plumaje que combinan tonalidades marrones, negras, gris y blanco, la morfología y distribución de las plumas en la mayoría de las aves es de característica normal (lisa típica), con distribución uniforme a lo largo del cuerpo, aunque se pueden encontrar ejemplares con patrones de plumaje diferente. Conclusiones. Se pudo observar una amplia variación entre animales procedentes de las 10 localidades estudiadas. Se evidencia una diferenciación tipológica con relación al peso y al tamaño del tarso, en los animales evaluados, lo que puede ser utilizado como criterio de selección.

Palabras clave: Avicultura, características fanerópticas, conservación, morfometría, recurso genético (Fuente: CAB).

\section{ABSTRACT}

Objective. Characterize morphometric and phaneroptics of the Creole backyard chickens from the savanna subregion department of Sucre (Colombia). Materials and methods. 520 adult Creole animals (350 chickens and 170 roosters) were evaluated, in the period 2017-2018, located in 10 localities of the savanna sub-region of the department of Sucre Colombia. The information was collected in a survey format composed of quantitative and qualitative variables. The data obtained were subjected to univariate descriptive analysis, for independent samples. In addition, the analysis of variance technique was used. Comparisons between males and females were made by means of a t-test. The analyses were performed using the statistical package R. Results. The descriptors analyzed showed the superiority of the roosters over the hens $(p<0.05)$. The analysis of variance in the population of females with respect to weight determined that there are significant differences $(p<0.05)$. The morphological and phaneroptic characteristics studied describe a yellow metatarsal bird, with plumage color that combines brown, black, gray and white tones, the morphology and distribution of feathers in most birds is normal characteristic (smooth typical), with a uniform distribution to a length of the body, although specimens with different plumage patterns can be found. Conclusions. A wide variation could be observed among animals from the 10 localities studied. There is evidence of a typological differentiation in relation to the weight and size of the tarsus in the animals evaluated, which can be used as a selection criterion.

Keywords: Conservation, genetic resources, morphometric, phaneroptic characteristics, poultry farming (Source: $C A B$ ).

Como citar (Vancouver)

Montes VD, de la Ossa VJ, Hernández HD.Caracterización morfológica de la gallina criolla de traspatio de la subregión Sabana departamento de Sucre (Colombia). Rev MVZ Cordoba. 2019; 24(2):7218-7224. DOI: https://doi.org/10.21897/rmvz.1646

(CEI (los) autor (es), Revista MVZ Córdoba 2019. Este artículo se distribuye bajo los términos de la licencia internacional Creative Commons Attribution 4.0 (https://creativecommons.org/licenses/by-sa/4.0/), que permite el uso sin restricciones, la distribución y la reproducción en cualquier medio, siempre que se otorgue el crédito apropiado al autor o autores originales y la fuente. 


\section{INTRODUCCIÓN}

La gallina (Gallus gallus domesticus) es una especies con distribución mundial, la cual no fue domesticada en el cercano o en el mediano oriente. Evidencias arqueológicas encontradas en el valle del Indo (Pakistán) y en la provincia china de Hebei, sugieren que esta especie fue domesticada a partir del gallo rojo selvático alrededor del año 74008000 años $(1,2)$, expandiéndose hacia Europa occidental, posiblemente, a través de Rusia. La domesticación puede haber ocurrido separadamente en India o haber sido introducida a través del sur de Asia $(3,4,5,6)$.

Se han propuesto dos hipótesis del proceso de formación de la gallina doméstica; la primera de ellas de origen monofilético, mientras que la segunda sostiene un origen múltiple a partir de varias subespecies de Gallus gallus que podrían ser considerados como posibles progenitores $(1,3)$. Sin embargo las razas más importantes surgieron durante la segunda mitad del siglo XIX (White Leghom, New Hampshire y Phymouth Rock) (7).

Existen varias teorías de la llegada de la gallina a América, una de ellas afirma que las gallinas llegaron al continente américano durante los primeros viajes de Cristóbal Colón (6); otra afirma que llegaron a América del Sur desde la isla de Pascua, ubicada al sudeste del océano Pacífico e introducidas al continente por pobladores provenientes de Polinesia (8).

Existen diferentes tipos de avicultura; dentro de ellas, la avicultura de gallinas criollas reviste importancia zootécnica no por la producción de huevos para el mercado, pues sus producciones anuales son muy bajas $(6,9)$, su racionalidad radica en constituirse en fuente importante de alimento de las familias campesinas, aporta a la base económica de la familia y es un recurso zoogenético importante (10). El concepto de gallina criolla según Tovar et al (11), es aquella gallina propia del lugar donde ha desarrollado sus características para su supervivencia; es bien reconocido, que sobre ellas se ha desarrollado cruzamiento constante desde antes del descubrimiento de América, ya sea por sí mismas o por intervención del hombre $(6,9)$.

La avicultura de traspatio, conocida como rural o criolla, constituye un sistema tradicional de producción pecuaria que realizan las familias campesinas en el patio de sus viviendas o alrededor de las mismas, se alimentan con insumos producidos por los propios campesinos o lo que ellas comen por si mismas en el campo y de desechos de la unidad familiar (7). Los problemas que enfrentan los recursos genéticos aviares criollos en la actualidad son la disminución de la variabilidad genética dentro de razas, la rápida desaparición de estirpes locales y líneas de animales domésticos a través de la introducción de razas exóticas, los climas cálidos, húmedos y otros ambientes hostiles. Es así como la conservación de razas naturales puras, tanto en el medioambiente silvestre como en el protegido, surge como alternativa para desarrollar diversos estudios, los cuales deben presentar como líneas base la identificación y caracterización de las variedades criollas explotadas, maximizando su variabilidad genética, considerando que, la sustitución de razas autóctonas por líneas comerciales constituye una amenaza real a los recursos genéticos avícolas locales. El presente estudio tuvo por objetivo la caracterización morfométrica y faneróptica la gallina criolla traspatio de la subregión Sabana, departamento de Sucre (Colombia).

\section{MATERIAL Y MÉTODOS}

Localización. Se caracterizaron 520 animales adultos criollos (350 gallinas y 170 gallos), en el periodo 20172018, ubicados en 10 localidades de la subregión Sabanas del departamento de Sucre (Sincelejo, Sincé, El Roble, San Pedro, Sampués, Los Palmitos, Galeras, Buenavista, Corozal y San Juan de Betulia) (Figura 1). El clima de la zona de estudio, es característico de las zonas de bosque seco tropical, persisten relictos de vegetación secundaria; se dan rastrojos y extensas áreas de pastizales. La temperatura promedio anual es de $27.2^{\circ} \mathrm{C}$, con precipitaciones fluctuantes ente 900 y 1.275 $\mathrm{mm}$ anuales y la humedad relativa del $80 \%(12,13)$. Las unidades de producción seleccionadas por municipio, realizan usualmente manejo tradicional para la cría de estirpe criolla (Gallus gallus domesticus - Phasianidae).

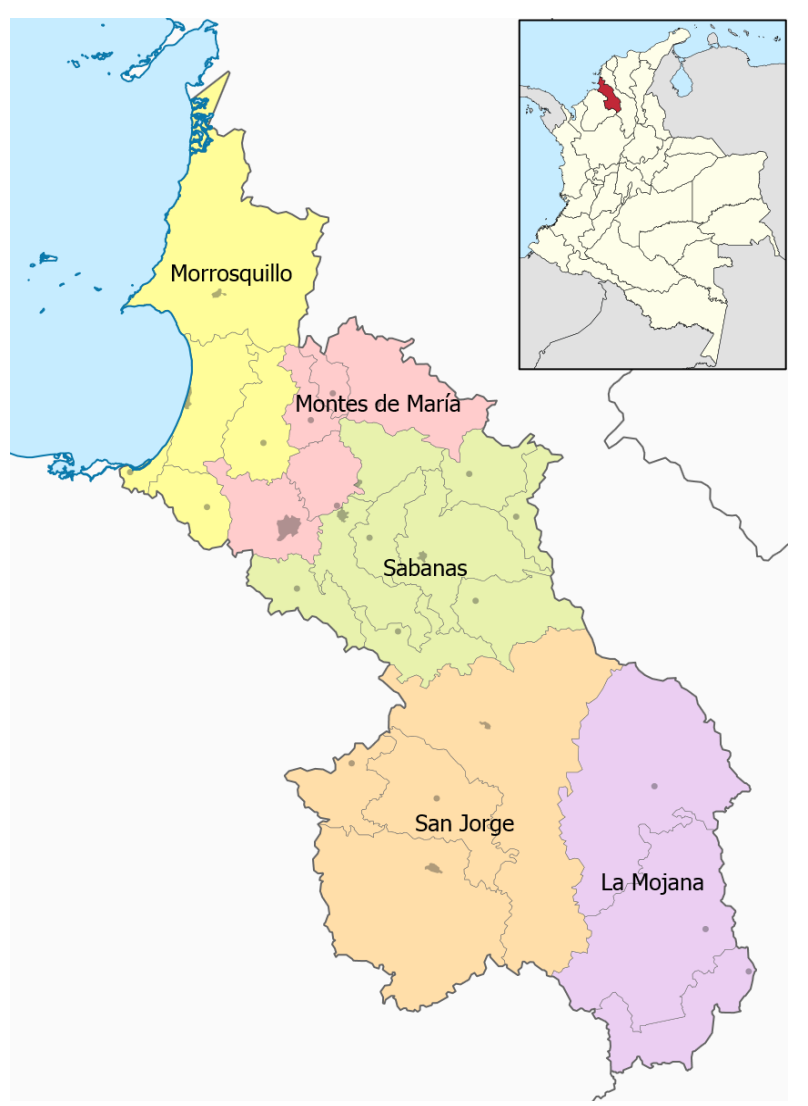

Figura 1. Ubicación zona de estudio, subregión sabana, departamento de Sucre Colombia.

Fuente: wikimedia.org

Se recolectó la información en un formulario, el cual integraba variables cuantitativas y cualitativas. Para la caracterización, se examinó la totalidad de las gallinas y gallos adultos encontrados en la unidad, solo fueron seleccionados aquellos animales en edad reproductiva.

Caracterización morfométrica. Los descriptores morfométricos evaluados correspondieron a 14 variables cuantitativas sugerida por la FAO (14), para la caracterización del recurso avícola y adaptadas por Lázaro et al (15) y Villacís et al (16). Para el análisis morfológico se consideraron los siguientes descriptores: peso $(\mathrm{kg})$, largo corporal, perímetro torácico, largo del muslo, largo 
de la pierna, largo del tarso, largo y ancho del ala, altura de la cresta, largo y ancho de la orejuela, largo y ancho de la barbilla y largo de cola; las medidas de longitud se expresaron en centímetros.

También se analizaron las siguientes características fanerópticas: morfología de la pluma, distribución del plumaje, patrón del plumaje, color del tarso, tamaño de la cresta y color del lóbulo de la oreja. Por otra parte se seleccionaron dos descriptores importantes que según Villacís et al (16) y Chicoya et al (17), tienen relación directa con la diferenciación morfológica de las gallinas y fueron el peso corporal $(\mathrm{g})$ y el largo del tarso $(\mathrm{cm})$.

Análisis de los datos. Las aves fueron pesadas y medidas empleando una balanza digital de mesa con capacidad de $6 \mathrm{~kg}$ y $0.001 \mathrm{~g}$ de resolución, una cinta métrica y un escalímetro con $0.03 \mathrm{~mm}$ de precisión.
Los datos obtenidos fueron sometidos a análisis descriptivos univariados para muestras independientes. Adicionalmente se empleó la técnica de análisis de varianza. Las comparaciones entre machos y hembras se realizaron mediante una prueba de $t$. Los análisis fueron realizados usando paquete estadístico $\mathrm{R}$ (18).

\section{RESULTADOS}

En las tablas 1 y 2 , se muestran los resultados promedio de las medidas morfométricas registradas en gallinas y gallos criollos. En todas las zonas los resultados de los valores medios observados de los descriptores analizados, mostraron superioridad de los gallos sobre las gallinas $(p<0.05)$.

Tabla1. Medidas morfométricas en gallinas biotipos criollos en comunidades de la subregión sabana del departamento de Sucre Colombia.

\begin{tabular}{|c|c|c|c|c|c|c|c|c|c|c|}
\hline \multirow[b]{2}{*}{ Parámetro } & \multicolumn{10}{|c|}{ Comunidades } \\
\hline & $\begin{array}{c}\text { Sincelejo } \\
n=30\end{array}$ & $\begin{array}{l}\text { Sincé } \\
n=38\end{array}$ & $\begin{array}{c}\text { El Roble } \\
n=36\end{array}$ & $\begin{array}{c}\text { San Pedro } \\
n=38\end{array}$ & $\begin{array}{l}\text { Los Palmitos } \\
\qquad n=\mathbf{3 8}\end{array}$ & $\begin{array}{c}\text { Sampués } \\
n=30\end{array}$ & $\begin{array}{c}\text { Corozal } \\
n=35\end{array}$ & $\begin{array}{c}\text { Galera } \\
n=32\end{array}$ & $\begin{array}{c}\text { S de Betulia } \\
n=35\end{array}$ & $\begin{array}{c}\text { Buena Vista } \\
n=\mathbf{3 8}\end{array}$ \\
\hline Peso (kg) & $2.20 \pm 0.5$ & $2.35 \pm 0.2$ & $1.8 \pm 0.3$ & $2.10 \pm 0.5$ & $1.90 \pm 0.4$ & $2.30 \pm 0.1$ & $2.0 \pm 0.1$ & $1.6 \pm 0.38$ & $1.8 \pm 0.5$ & $1.9 \pm 0.2$ \\
\hline Largo corporal (cm) & $40.4 \pm 6.8$ & $35.5 \pm 3.4$ & $42.9 \pm 4.0$ & $45.7 \pm 3.7$ & $43.5 \pm 5.8$ & $38.7 \pm 6.7$ & $39.4 \pm 2.7$ & $36.2 \pm 3.9$ & $35.6 \pm 5.5$ & $39.3 \pm 3.6$ \\
\hline P-Torácico(cm) & $30.6 \pm 2.9$ & $35.5 \pm 1.5$ & $32.9 \pm 4.1$ & $35.7 \pm 2.8$ & $39.5 \pm 2.9$ & $38.7 \pm 3.8$ & $35.4 \pm 1.8$ & $33.2 \pm 4.5$ & $39.6 \pm 2.6$ & $34.3 \pm 2.7$ \\
\hline Largo muslo(cm) & $9.2 \pm 0.8$ & $9.0 \pm 2.6$ & $11.0 \pm 0.2$ & $10.1 \pm 1.3$ & $10.3 \pm 1.2$ & $9.5 \pm 0.58$ & $10.5 \pm 1.8$ & $11.1 \pm 2.5$ & $12.2 \pm 0.5$ & $9.8 \pm 2.6$ \\
\hline Largo Pierna (cm) & $11.5 \pm 1.9$ & $8.5 \pm 1.0$ & $8.4 \pm 2.3$ & $9.2 \pm 1.9$ & $10.4 \pm 2.8$ & $11.5 \pm 1.4$ & $10.9 \pm 1.9$ & $12.1 \pm 1.3$ & $11.8 \pm 1.5$ & $10.9 \pm 2.6$ \\
\hline Largo de tarso $(\mathrm{cm})$ & $9.1 \pm 0.3$ & $8.9 \pm 1.4$ & $9.6 \pm 0.2$ & $9.1 \pm 1.6$ & $8.1 \pm 1.7$ & $8.1 \pm 2.8$ & $10.1 \pm 1.3$ & $9.3 \pm 0.9$ & $10.0 \pm 1.6$ & $9.2 \pm 1.6$ \\
\hline Largo ala $(\mathrm{cm})$ & $27.8 \pm 1.1$ & $30.5 \pm 2.9$ & $28 \pm 4.5$ & $30.3 \pm 3.8$ & $29.2 \pm 1.8$ & $29.0 \pm 2.5$ & $29.2 \pm 2.1$ & $27.8 \pm 4.4$ & $30.3 \pm 0.8$ & $31.1 \pm 2.4$ \\
\hline Largo orejuela $(\mathrm{cm})$ & $2.25 \pm 0.6$ & $1.92 \pm 0.3$ & $1.84 \pm 0.4$ & $1.53 \pm 0.5$ & $1.94 \pm 0.5$ & $1.93 \pm 0.1$ & $2.04 \pm 0.2$ & $1.66 \pm 0.5$ & $1.83 \pm 0.2$ & $1.94 \pm 0.2$ \\
\hline Largo barbillas (cm) & $1.3 \pm 0.13$ & $1.15 \pm 0.3$ & $1.1 \pm 0.5$ & $1.90 \pm 0.9$ & $1.90 \pm 0.5$ & $1.1 \pm 0.2$ & $1.2 .0 \pm 0.5$ & $1.6 \pm 0.4$ & $1.8 \pm 0.6$ & $1.3 \pm 0.6$ \\
\hline Largo cola $(\mathrm{cm})$ & $18.5 \pm 1.4$ & $17.5 \pm 2.2$ & $16.5 \pm 2.8$ & $18.4 \pm 2.4$ & $19.0 \pm 0.9$ & $16.80 \pm 1.3$ & $18.4 \pm 0.9$ & $1.7 \pm 2.5$ & $16.9 \pm 3.5$ & $19.5 \pm 1.2$ \\
\hline Ancho ala $(\mathrm{cm})$ & $22.2 \pm 1.8$ & $23.5 \pm 0.3$ & $18.5 \pm 2.9$ & $21.2 \pm 1.1$ & $18.5 \pm 0.1$ & $19.1 \pm 2.3$ & $20.5 \pm 2.5$ & $16.9 \pm 2.9$ & $18.6 \pm 1.2$ & $19.2 \pm 2.8$ \\
\hline Ancho orejuela $(\mathrm{cm})$ & $1.20 \pm 0.3$ & $1.36 \pm 0.5$ & $1.82 \pm 0.8$ & $1.71 \pm 0.6$ & $1.80 \pm 0.4$ & $1.75 \pm 0.2$ & $1.77 \pm 0.6$ & $1.7 \pm 0.9$ & $1.6 \pm 0.8$ & $1.8 \pm 0.3$ \\
\hline Ancho barbilla $(\mathrm{cm})$ & $2.20 \pm 0.1$ & $2.05 \pm 0.3$ & $1.6 \pm 0.5$ & $2.10 \pm 0.3$ & $2.09 \pm 0.5$ & $2.18 \pm 0.2$ & $1.8 \pm 0.3$ & $1.6 \pm 0.6$ & $2,53 \pm 0.4$ & $2.03 \pm 0.4$ \\
\hline Altura cresta $\mathrm{Cm}$ ) & $1.89 \pm 0.1$ & $1.68 \pm 0.3$ & $1.8 \pm 0.3$ & $1.9 \pm 0.3$ & $1.98 \pm 0.2$ & $2.30 \pm 0.3$ & $2.39 \pm 0.2$ & $2.12 \pm 0.5$ & $1.85 \pm 0.7$ & $1.91 \pm 0.8$ \\
\hline
\end{tabular}

Tabla 2. Medidas morfométricas en gallos biotipos criollos en comunidades de la subregión sabana del departamento de Sucre Colombia.

\begin{tabular}{|c|c|c|c|c|c|c|c|c|c|c|}
\hline \multirow[b]{2}{*}{ Parámetro } & \multicolumn{10}{|c|}{ Comunidades } \\
\hline & $\begin{array}{c}\text { Sincelejo } \\
n=12\end{array}$ & Sincé $n=15$ & $\begin{array}{c}\text { EI Roble } \\
n=15\end{array}$ & $\begin{array}{c}\text { San Pedro } \\
n=18\end{array}$ & $\begin{array}{c}\text { Los Palmitos } \\
n=19\end{array}$ & $\begin{array}{c}\text { Sampués } \\
n=23\end{array}$ & $\begin{array}{c}\text { Corozal } \\
n=21\end{array}$ & $\begin{array}{c}\text { Galera } \\
n=12\end{array}$ & $\begin{array}{c}\text { S de Betulia } \\
n=15\end{array}$ & $\begin{array}{c}\text { Buena Vista } \\
\mathbf{n}=\mathbf{2 0}\end{array}$ \\
\hline Peso (kg) & $2.90 \pm 0.3$ & $2.45 \pm 1.3$ & $2.8 \pm 0.3$ & $2.70 \pm 1.5$ & $2.90 \pm 0.9$ & $3.10 \pm 0.1$ & $2.60 \pm 1.5$ & $2.7 \pm 1.2$ & $2.8 \pm 1.2$ & $2.9 \pm 0.61$ \\
\hline Largo corporal (cm) & $44.4 \pm 1.8$ & $49.6 \pm 2.9$ & $45.4 \pm 2.1$ & $38.4 \pm 1.1$ & $40.2 \pm 3.1$ & $43.2 \pm 2.1$ & $39.3 \pm 3.3$ & $36.4 \pm 2.1$ & $40.4 \pm 2.1$ & $38.4 \pm 3.1$ \\
\hline P- torácico $(\mathrm{cm})$ & $38.6 \pm 1.9$ & $37.5 \pm 3.5$ & $35.9 \pm 2.1$ & $39.7 \pm 3.8$ & $38.5 \pm 1.9$ & $41.7 \pm 2.8$ & $39.4 \pm 1.8$ & $35.2 \pm 4.5$ & $37.6 \pm 2.6$ & $36.3 \pm 2.7$ \\
\hline Largo muslo(cm) & $12.2 \pm 1.7$ & $12.5 \pm 1.2$ & $11.5 \pm 1.7$ & $11.2 \pm 1.2$ & $12.5 \pm 2.1$ & $14.2 \pm 0.5$ & $13.2 \pm 1.6$ & $12.4 \pm 1.1$ & $11.5 \pm 1.57$ & $11.2 \pm 2.8$ \\
\hline Largo Pierna (cm) & $15.5 \pm 1.4$ & $14.3 \pm 2.1$ & $14.0 \pm 2.6$ & $16.2 \pm 1.7$ & $16.5 \pm 0.8$ & $17.2 \pm 0.9$ & $13.9 \pm 2.1$ & $14.3 \pm 3.5$ & $15.2 \pm 2.1$ & $16.5 \pm 1.8$ \\
\hline Largo de tarso(cm) & $9.5 \pm 1.3$ & $9.8 \pm 2.6$ & $8.1 \pm 2.5$ & $10.1 \pm 1.6$ & $8.1 \pm 2.7$ & $10.1 \pm 1.5$ & $10.1 \pm 2.3$ & $9.3 \pm 0.9$ & $9.0 \pm 1.7$ & $10.2 \pm 2.6$ \\
\hline Largo ala $(\mathrm{cm})$ & $33.2 \pm 3.1$ & $33.5 \pm 3.9$ & $35.2 \pm 2.5$ & $36.3 \pm 4.8$ & $39.2 \pm 1.8$ & $37.0 \pm 3.5$ & $34.2 \pm 1.1$ & $37.8 \pm 2.4$ & $36.3 \pm 1.5$ & $36.1 \pm 3.2$ \\
\hline Largo orejuela (cm) & $2.15 \pm 1.6$ & $2.92 \pm 1.3$ & $2.84 \pm 0.8$ & $3.13 \pm 0.2$ & $2.94 \pm 1.5$ & $3.03 \pm 0.3$ & $3.04 \pm 0.9$ & $2.96 \pm 3.3$ & $3.13 \pm 1.1$ & $2.94 \pm 2.3$ \\
\hline Largo barbillas (cm) & $3.3 \pm 0.13$ & $4.15 \pm 0.3$ & $3.5 \pm 1.57$ & $3.90 \pm 1.9$ & $4.10 \pm 0.3$ & $3.8 \pm 0.59$ & $4.20 \pm 0.5$ & $3.6 \pm 2.4$ & $3.8 \pm 2.62$ & $3.3 \pm 3.6$ \\
\hline Largo cola $(\mathrm{cm})$ & $28.5 \pm 1.4$ & $26.5 \pm 2.4$ & $26.5 \pm 4.8$ & $28.4 \pm 5.4$ & $29.0 \pm 0.9$ & $26.80 \pm 3.3$ & $24.4 \pm 2.9$ & $27.7 \pm 2.5$ & $26.9 \pm 3.5$ & $28.5 \pm 1.3$ \\
\hline Ancho ala (cm) & $25.2 \pm 1.8$ & $24.5 \pm 0.3$ & $28.5 \pm 2.9$ & $26.2 \pm 1.2$ & $28.5 \pm 2.1$ & $25.1 \pm 2.3$ & $26.5 \pm 2.9$ & $26.9 \pm 2.3$ & $24.6 \pm 4.2$ & $29.2 \pm 2.8$ \\
\hline Ancho orejuela(cm) & $1.70 \pm 1.3$ & $2.70 \pm 0.3$ & $1.60 \pm 1.3$ & $1.80 \pm 1.3$ & $2.1 \pm 1.4$ & $2.20 \pm 1.4$ & $2.3 \pm 1.42$ & $1.70 \pm 1.4$ & $1.60 \pm 2.4$ & $1.90 \pm 3.4$ \\
\hline Ancho barbilla $(\mathrm{cm})$ & $4.20 \pm 1.1$ & $4.05 \pm 2.3$ & $4.61 \pm 1.5$ & $4.20 \pm 0.32$ & $4.3 \pm 0.5$ & $4.18 \pm 3.2$ & $4.5 \pm 0.5$ & $4.0 \pm 3.5$ & $4,33 \pm 0.4$ & $4.13 \pm 0.8$ \\
\hline Altura cresta $\mathrm{Cm}$ ) & $3.89 \pm 1.1$ & $5.08 \pm 0.3$ & $4.8 \pm 1.5$ & $4.320 \pm 2.1$ & $3.98 \pm 3.2$ & $4.30 \pm 2.2$ & $5.39 \pm 0.2$ & $4.12 \pm 0.5$ & $4.85 \pm 0.7$ & $5.11 \pm 0.8$ \\
\hline
\end{tabular}


El análisis de varianza en la población de hembras con respecto al peso determinó que existen diferencias $(p<0.05)$, estableciéndose tres grupos de gallinas: pesadas, livianas y semipesadas (Tabla 3). De igual forma se estableció la agrupación de machos en dos conglomerados (machos pesados y machos livianos) respectivamente.

Tabla 3. Variables morfométricas de diferenciación morfológica de biotipo criollo de gallina y gallos en comunidades de la subregión sabana del departamento de Sucre Colombia.

\begin{tabular}{ccc}
\hline \multicolumn{3}{c}{ Gallinas } \\
\hline Grupo & Peso(gr) & Largo del tarso $(\mathbf{c m})$ \\
\hline $\mathbf{1}$ & $2586^{\mathrm{a}}$ & $9.45^{\mathrm{a}}$ \\
$\mathbf{2}$ & $1260^{\mathrm{b}}$ & $7.8^{\mathrm{b}}$ \\
$\mathbf{3}$ & $1678^{\mathrm{c}}$ & $8.32^{\mathrm{a}}$ \\
& Gallos & \\
$\mathbf{1}$ & $3123^{\mathrm{a}}$ & $12.15^{\mathrm{a}}$ \\
$\mathbf{2}$ & $2566^{\mathrm{b}}$ & $8.82^{\mathrm{b}}$ \\
\hline
\end{tabular}

$a, b, c$ : letras dentro de columnas indican diferencia estadística $(p<0.001)$ Grupo: peso: 1: pesada; 2: liviana; 3: semipesada

Largo de tarso: 1: grande; 2: pequeña; 3: mediana (Villacís et al (15))

Con relación a la variable tamaño del tarso, se diferenciaron para gallinas y gallos 2 grupos, un de animales grandes y otro de animales medianos (Tabla 3 ).

En la tabla 4, se observan los resultados del análisis de las características fanerópticas. Se evidenció, un estándar de gallina y de gallo con dominancia de plumaje con característica normal, con una frecuencia del $90 \%$ y $95 \%$ respectivamente. La distribución del plumaje es mayoritariamente de característica normal (gallinas $77 \%$ y gallos $75 \%$ ) y el patrón del plumaje es uniforme en un $66 \%$. Existen colores básicos que predominan en el plumaje de las gallinas y gallos como son: el color marrón, con una frecuencia del $38 \%$ y $31 \%$, luego el negro con $23.16 \%$, el gris con $14.76 \%$ y el blanco con $11.70 \%$. Sin embargo, estos colores se combinan con otros colores de pluma, principalmente en alas, dorso y cola (Figura 2).

Con respecto al color del tarso, el $51 \%$ de las gallinas y $85 \%$ de los gallos, presentaron coloración amarillenta, seguidas por coloraciones blancas y negras en las hembras y verdes en los machos.

En las características de la cabeza se determinó que las gallinas presentan un tamaño de cresta que oscila entre ligeramente pequeña y mediana (43 y 53\% respectivamente), con tres a cuatro dientes bien definidos. Los gallos en tanto presentaron un tamaño que osciló entre mediano a grande en proporciones de 29 a $71 \%$ respectivamente.

Las orejuelas de tamaño moderado y ovaladas, bien pegadas a la cara y pigmentada (roja $65 \%$ y roja-blanca $34 \%$ ). El color de ojos dominante es el rojo - naranja, con una frecuencia de 79 y $92 \%$ en hembras y machos, respectivamente.
Tabla 4. Frecuencias absolutas y relativas de características fanerópticas de gallinas biotipos criollos en comunidades de la subregión sabana del departamento de Sucre Colombia.

\begin{tabular}{|c|c|c|c|c|}
\hline \multirow{2}{*}{ Variables cualitativas } & \multicolumn{2}{|c|}{ Hembras } & \multicolumn{2}{|c|}{ Machos } \\
\hline & $\mathbf{F R}$ & FA & $\mathbf{F R}$ & FA \\
\hline \multicolumn{5}{|c|}{ Morfología pluma } \\
\hline Normal & 314 & 0.9 & 162 & 0.95 \\
\hline Rizada & 33 & 0.09 & 8 & 0.05 \\
\hline Sedosa & 3 & 0.01 & 0 & 0 \\
\hline \multicolumn{5}{|c|}{ Distribución del plumaje } \\
\hline Normal & 269 & 0.77 & 124 & 0.73 \\
\hline Cuello desnudo & 60 & 0.17 & 40 & 0.24 \\
\hline Patas y/o Tarzos emplumados & 21 & 0.06 & 6 & 0.04 \\
\hline \multicolumn{5}{|c|}{ Patrón del plumaje } \\
\hline Uniforme & 267 & 0.76 & 112 & 0.66 \\
\hline Crespo & 2 & 0.01 & 2 & 0.01 \\
\hline Barrado & 58 & 0.17 & 55 & 0.32 \\
\hline Jaspeado & 15 & 0.04 & 1 & 0.01 \\
\hline Moteado & 6 & 0.02 & 0 & 0 \\
\hline Otros & 2 & 0.01 & 0 & 0 \\
\hline \multicolumn{5}{|c|}{ Color del plumaje } \\
\hline Blanco & 52 & 0.15 & 29 & 0.17 \\
\hline Negro & 77 & 0.22 & 43 & 0.26 \\
\hline Cenizo( azulado) & 56 & 0.16 & 32 & 0.19 \\
\hline Marrón & 133 & 0.38 & 52 & 0.31 \\
\hline Otros & 32 & 0.09 & 14 & 0.09 \\
\hline \multicolumn{5}{|c|}{ Color del tarso } \\
\hline Verdoso & 30 & 0.09 & 16 & 0.09 \\
\hline Blanco & 78 & 0.22 & 4 & 0.02 \\
\hline Amarillo & 180 & 0.51 & 144 & 0.85 \\
\hline Azul & 0 & 0 & 0 & 0 \\
\hline Negro & 50 & 0.14 & 4 & 0.02 \\
\hline Marrón & 12 & 0.03 & 2 & 0.01 \\
\hline
\end{tabular}

Color del lóbulo de la oreja

\begin{tabular}{ccccc} 
No pigmentado( Blanco) & 2 & 0.01 & 1 & 0.01 \\
Rojo & 228 & 0.65 & 94 & 0.55 \\
Rojo-Blanco & 120 & 0.34 & 75 & 0.44 \\
& Tamaño de la cresta & & & \\
Pequeña & 150 & 0.43 & 1 & 0.01 \\
Mediana & 185 & 0.53 & 49 & 0.29 \\
Grande & 15 & 0.04 & 120 & 0.71 \\
& Color del ojo & & & \\
Negro & 12 & 0.03 & 2 & 0.01 \\
Rojo-Naranja & 278 & 0.79 & 156 & 0.92 \\
Perla & 44 & 0.13 & 12 & 0.07 \\
Marrón & 16 & 0.05 & 0 & 0 \\
\hline
\end{tabular}



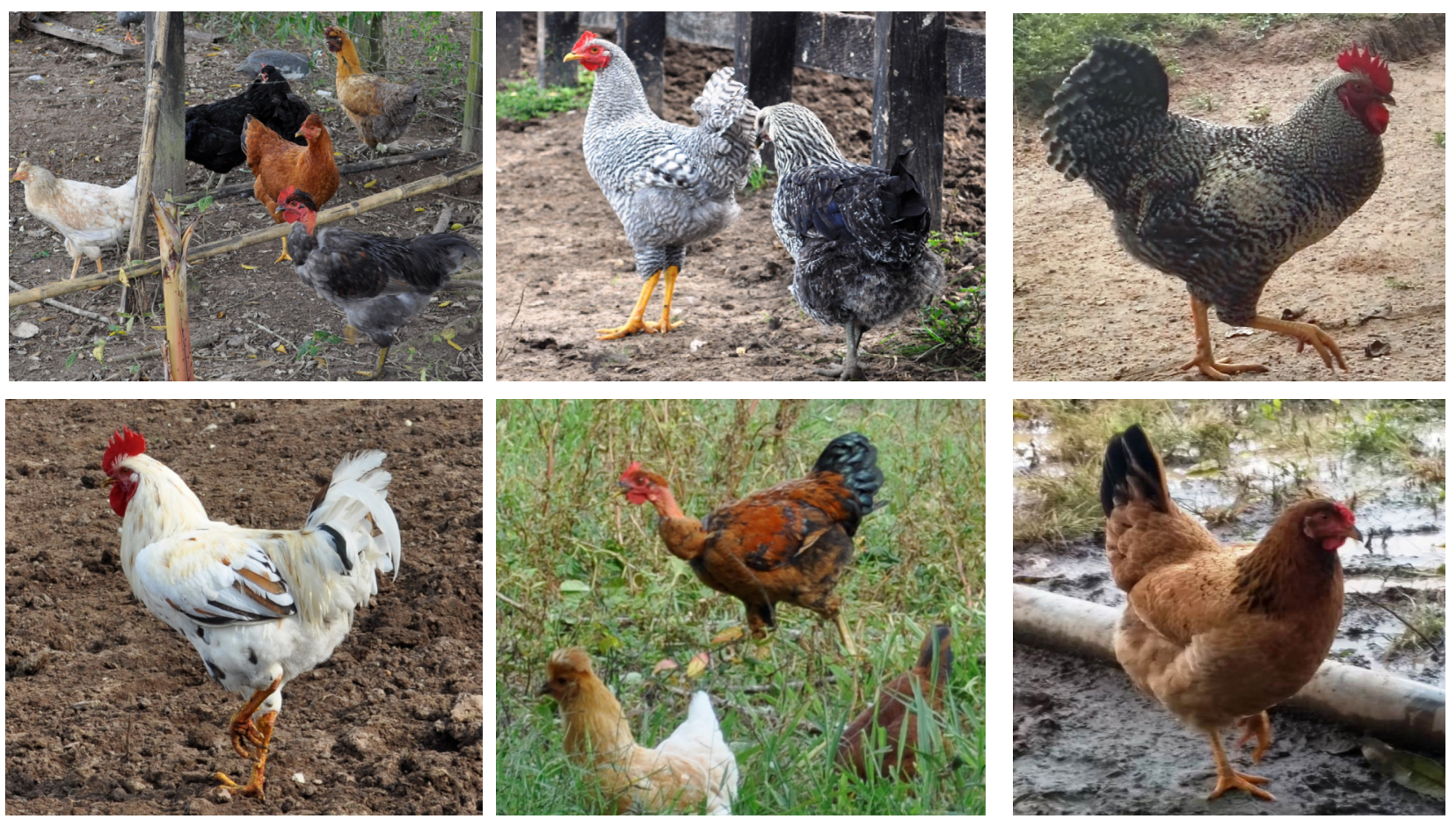

Figura 2. Biotipos criollos de Gallus gallus domesticus (Phasianidae), Subregión sabana, departamento de Sucre Colombia.

\section{DISCUSIÓN}

Las variables peso, perímetro pectoral, largos de tarso y largos de muslo, en conjunto, definen la talla o complexión del ave, que refleja el propósito de la crianza de las gallinas en las unidades de producción. En ese sentido, las medidas zoométricas encontradas en los animales estudiados (Tabla 1 y 2), explican el tamaño y talla del animal, lo que constituye la base para establecer los descriptores fenotípicos como criterios de tipología o agrupamiento de las aves, lo que da lugar a tener tres agrupaciones de gallinas y a dos de gallos (Tabla 3 ).

El primer grupo de aves que se caracterizó como pesadas, mayores de $2.5 \mathrm{~kg}$; por consiguiente, poseen extremidades más grandes, como es el tarso y largo de pierna, las cuales son criadas en las unidades de producción con la finalidad de obtener huevos y carne al final de la vida productiva (16).

Un segundo grupo fue identificado como liviano, con un peso menor a $1.3 \mathrm{~kg}$, caracterizado por poseer características de menor tamaño. Esto sugiere que la finalidad de estas aves en la unidad de producción es de postura, con las cuales se obtienen una mayor cantidad de huevos en cada ciclo. Estos resultados fueron similares a los reportados por Villacís et al (16), Vega (19) y Jáuregui et al (20).

Un tercer grupo de aves; que fue el predominante, presentó un peso menor a $1.700 \mathrm{~kg}$ y tamaño mediano; evidenciando que son animales con una actitud de doble finalidad, siendo preferidas para la obtención de huevo (16).

Las medidas corporales de los machos son mayores $(p<0.05)$ que las de las hembras $(21)$. En promedio las medidas de las hembras son $10 \%$ menores que los machos y en algunos casos, como en el tamaño de la cresta la diferencia es alrededor del $200 \%$. Resultados similares de medidas zoométricas de Biotipos de gallinas y de gallos criollos fueron reportados por Lázaro et al (15), demostrando así que en las aves estudiadas no existe homogeneidad, lo que facilita la variabilidad y concomitantemente la creación de líneas productivas rusticas y adaptadas trópico que contribuyen con la conservación de sistemas de producción tradicionales y socialmente sustentables para los pobladores del campo.

Se realizó una comparación de proporciones, mediante la prueba de Chi-cuadrado, contrastándose el color de la pluma, morfología de la pluma, patrón del plumaje, distribución del plumaje, tamaño de cresta, color del tarso, color de las orejuelas y color del ojo. Las características morfológicas y fanerópticas estudiadas, describen un ave de metatarso amarillo, con color de plumaje que combinan tonalidades marrones, negras, gris y blanco (20), la morfología y distribución de las plumas en la mayoría de las aves es de característica normal, con distribución uniforme a lo largo del cuerpo, aunque se pueden encontrar ejemplares con patrones de plumaje diferente (Tabla 4).

En general las gallinas presentan cabeza alargada, delgada y fina, poseen cresta de tamaño que oscilan entre mediana a pequeña, con color de orejuelas entre tonalidades de rojo-blanco y ojos generalmente de color rojo-a naranja. Por el contrario, los gallos presentan en la cabeza crestas de tamaño grande. El tamaño y forma de la cabeza, se encuentra relacionada directamente con el dimorfismo sexual del ave (22), en los ejemplares machos son desarrollados y vistosos, por el contrario en hembras, son de poco tamaño. 
La apariencia fenotípica de ejemplares observados, corresponde en algunos casos, a estirpes comerciales, evidenciándose el grado de penetración de aves de razas introducidas, a la población de gallinas criollas del área. Los animales de emplume de característica normal muestran una alta frecuencia, lo que permite asumir, mayor capacidad de adaptación a las condiciones climáticas, aunque esta característica es provocada por la acción de genes recesivos (23).

En conclusión, se pudo observar una amplia variación entre animales procedentes de las 10 localidades estudiadas en este estudio. Tomando como base dos descriptores principales como son el peso del animal y largo del tarso, las gallinas criollas pueden ser agrupadas bajo los siguientes criterios: gallinas pesadas (mayores de $2.5 \mathrm{~kg}$ ); gallinas semipesadas (entre 1.5 y $2.49 \mathrm{~kg}$ ); $y$, gallinas ligeras (menores de $1.5 \mathrm{~kg}$ ). Esta clasificación puede ser considerada como criterios de diferenciación tipológica y selección de reproductores criollos. Se deben implementar medidas para la conservación de la gallina criolla como recurso local, puesto que los resultados encontrados, reflejan una tendencia a la homogeneidad posibilitando la pérdida de la diversidad fenotípica de la parvada criolla.

\section{Conflicto de interés}

Los autores declaran no tener conflictos de interés

\section{REFERENCIAS}

1. Giovambattista G, Liron J, Bravi C, Prando A. El aporte de la Genética en la elucidación de la Historia de la Domesticación y Diferenciación de las Expecies Domésticas. En: Capitulo 5 [Internet]. Ciudad Autónoma de Buenos Aires: Inter-Médica; 2010 [citado 13 de marzo de 2019]. p. 73-98. Disponible en: https://www.conicet.gov.ar/new scp/detalle. php?keywords $=\&$ id $=19311$ \&capitulos $=$ yes \&detall es=yes\&capit id $=965996$

2. Balanoff A, Bever G, Rowe T, Norell $M$. Evolutionary origins of the avian brain. Nature. 2013;501(7465):93-6. DOI: https://10.1038/ nature 12424

3. Miao Y, Peng M, Wu G, Ouyang Y, Yang Z, Yu N, et al. Chicken domestication: an updated perspective based on mitochondrial genomes. Heredity. 2013; 110(3):277-82. DOI: http://10.1038/hdy.2012.83

4. Larson G, Fuller D. The Evolution of Animal Domestication. Annual Review of Ecology, Evolution, and Systematics. 2014; 45(1):115-36. DOI: http://10.1146/annurev-ecolsys-110512-135813

5. Xiang $\mathrm{H}$, Gao J, Yu B, Zhou H, Cai D, Zhang Y, et al. Early Holocene chicken domestication in northern China. PNAS. 2014; 111(49):17564-9. DOI: http:// doi.org/10.1073/pnas.1411882111

6. Palacios E, Álvarez L, Muñoz J. Genetic diversity of Creole hens of the Colombian southwest. Archivos de Zootecnia. 2016; 65(249):73-8. DOI: http://10.21071/az.v65i249.444

7. Correa J, Salas M, Franco L, Ricalde R. Indicadores de producción de huevo de gallinas criollas en el trópico de México. Archivos de Zootecnia. 2007; 56(215):309-17. URL Available in: https://dialnet. unirioja.es/servlet/articulo?codigo $=2381470$

8. Storey A, Rami J, Quiroz D, Burley D, Addisom D, Walter R, et al. Radiocarbon and DNA evidence for a pre-Columbian introduction of Polynesian chickens to Chile. PNAS. 2007; 104(25):10335-10339. DOI: http://doi10.1073pnas.0703993104
9. Valencia N. La gallina criolla colombiana [Internet]. Universidad Nacional de Colombia, Sede Palmira. Palmira, Colombia: UNIMEDIOS; 2011. 61 p. Disponible en: http://bdigital.unal.edu. co/3412/1/9789588095561.pdf

10. Enrique G, Sánchez E, Castillo F, Agusto R. Características fenotíicas de las gallinas criollas de comunidades rurales del sur de Ecuador. Centro de Biotecnología [Internet]. 2014 [citado 14 de marzo de 2019]; 3(1). Disponible en: http://revistas.unl. edu.ec/index.php/biotecnologia/article/view/30

11. Tovar J, Narváez-Solarte W, Takahashi S. Baes for the colombian Gallus gallus domesticus (Phasianidae) conservation inthe departament of Caldas. Boletin Cientifico Centro de Museos de Historia Natural Universiad de Caldas. 2014; 18(1):112-22. Disponible en: http://www.scielo. org.co/scielo.php?script=sci abstract\&pid=S012330682014000100007\&lng=en\&nrm=iso\&tlng=es

12. De la Ossa J, Botero L. Vías pecuarias e importancia en la trashumancia ganadera. Revista Colombiana de Ciencia Animal - RECIA. Revista Colombiana de Ciencia Animal. 2013; 5(1):431-8. Disponible en: https://revistas.unisucre.edu.co/index.php/recia/ article/view/456

13. Montes-Vergara D, De la Ossa J, Pérez-Cordero A. Determinación mediante prueba cometa del daño genético causado por uso de ivermectina en vacas cebú (Bos taurus indicus). Rev MVZ Cordoba. 2017; 22(2):5959-65. https://doi.org/10.21897/ rmvz.1034

14. FAO. 1981. Descriptores de especies avícolas. Banco de datos de recursos genéticos animales. Roma, Italia. vols. 13-15.

15. Lázaro G, Hernandez Z, Vargas L, Martínez L, Pérez A. Uso de caracteres morfométricos en la clasificación de gallinas locales. AICA. 2012; 2:10914. Disponible en: http://www.uco.es/conbiand/ aica/templatemo 110 lin photo/articulos/2012/ Trabajo049 AICA2012.pdf 
16. Villacís G, Escudero G, Cueva F, Luzuriaga A. Características morfométricas de las gallinas criollas de comunidades rurales del sur del Ecuador. Revista de Investigaciones Veterinarias del Perú. 2016; 27(2):218-24. DOI: http://10.15381/rivep. v27i2.11639

17. Chincoya H, Haro J, Salas M, Varela A, Garay A. Tipología de gallinas criollas en valles centrales Oaxaca con base en descriptores morfométricos. Agricultura Sociedad y Desarrollo. 2018; 15(4):58593. DOI: http://10.22231/asyd.v15i4.901

18. R: The R Project for Statistical Computing [Internet]. [citado 14 de marzo de 2019]. Disponible en: https://www.r-project.org/

19. Vega V, Roman S, Duran M, Velez A, Cabrera E, Cantu A, et al. Morphological evaluation Mexican backyard chickens (Gallus gallus domesticus). Revista Mexicana de Ciencias Pecuarias. 2018; 9(2):1-14. DOI: http://dx.doi.org/10.22319/rmcp. v9i2.4484

20. Jáuregui $R$, Flores $H$, Vasquez $L$, Oliva $M$. Caracterización morfométrica de la gallina de cuello desnudo (Gallus domesticus nudicollis) en la región ch'ortí de Chiquimula, Guatemala. Ciencia, Tecnología y Salud. 2015; 2(1):1-8. Disponible en: https://digi.usac.edu.gt/ojsrevistas/index.php/ cytes/article/viewFile/42/41
21. Zaragoza M, Rodríguez $H$, Hernández Z, Perezgrovas G, Martínez C, Méndez E. Caracterización de gallinas Batsi Alak en las tierras altas del sureste de México. Archivos de Zootecnia. 2013; 62(239):321-32. DOI: http://10.21071/az.v62i239.638

22. Negassa D, Melesse A, Banerjee S. Phenotypic characterization of indigenous chicken populations in Southeastern Oromia Regional State of Ethiopia. Animal Genetic Resources/ Resources génétiques animales/Recursos genéticos animales. 2014; 55:101-13. DOI: http://10.1017/ $\underline{\mathrm{S} 2078633614000319}$

23. Valdés RJ, Pimentel O, Martínez K, Ferro E. Caracterización fenotípica del genofondo avícola criollo de San Andrés, Pinar del Río, Cuba. Archivos de Zootecnia. 2010; 59(228):597-600. Disponible en: http://scielo.isciii.es/scielo.php?script $=$ sci abstract\&pid =S0004-05922010000400013\&lng = es\&nrm=iso\&tlng=es 